\title{
EL CONTROL DE LA NATALIDAD Y EL ABORTO PROVOCADO
}

\author{
Dr. Eduardo Acosta Bendek*
}

El aborto provocado como rechazo de la maternidad indeseada es tan antiguo como la misma historia de la humanidad, por cuanto en los pueblos primitivos el aborto criminal y el infanticidio se aceptaban socialmente; no sólo no eran sancionados, sino, considerados como actos naturales y comunes. A medida que la civilización fue alcanzando un gradó de mayor desarrollo moral, religioso y cultural, estas prácticas fueron condenadas gradualmente. En la antigua Grecia, Hipócrates en su juramento, condenó y rechazó la interrupción de la gestación. Al mismo tiempo, el Cristianismo defendía el derecho a la vida del feto y del niño. De otra parte, las culturas medioevales se preocuparon por el control de la fertilidad, como lo comprueba el hecho del uso de una variedad de preparaciones a base de plantas y de compuestos de origen vegetal y mineral con esos propósitos.

Superadas las prácticas anteriores y ya entrado el siglo XVIII, la alarmante morbi-mortalidad materna producida por el aborto criminal como control de la natalidad, se constituyó en un problema médico-social serio que obligó a países como Rusia, Japón, Suecia, Dinamarca y Hungría (1), entre otros, a legalizar el aborto ,tratando de conseguir con esas medidas reducir la alta morbi-mor- talidad materna al autorizar su práctica en medios quirúrgicos y con personal idóneo. Sin embargo, los ginecólogos rusos (2) después de una gran experiencia en este campo advierten que en cirugía ginecológica ninguna intervención tiene tantos riesgos como la del aborto provocado.

Después de haber transcurrido varias centurias y realizar grandes esfuerzos científicos se logró obtener, en este campo del control de la natalidad, un verdadero progreso como fue el descubrimiento de las hormonas sexuales (estrógeno - progesterona), hasta llegar, después de una larga serie de investigaciones, al descubrimiento de los ovulistáticos en sus distintas variedades, sin que se pueda afirmar aun que se ha obtenido o alcanzado el anticonceptivo ideal.

El propósito del presente estudio realizado en un grupo de pacientes cle condiciones socio-económicas, culturales y religiosas de bajo nivel, elaborado en la Maternidad "Leopoldina de Santos" del Hospital de Barranquilla, busca analizar los múltiples aspectos de este complejo problema médico-social desde el punto de vista clínico, económico, social y cultural.

* Jefe del Departamento de Obstetricia y Ginecología del Hospital de Barranquilla. 


\section{Material y Métodos}

Este estudio se llevó a cabo en la sección de abortos de la Maternidad "Leopoldina de Santos" del Hospital de Barranquilla, en donde se seleccionaron 700 casos en los que se obtuvo un mayor número de datos útiles, para lo cual se hizo impresión de una hoja especial adicional a la historia clínica con una clasificación del aborto en relación con el estado civil, alimentación, edad, raza, antecedentes obstétricos y quirúrgicos, grado de instrucción, situación económica, deseos de tener hijos, complementado con el estudio histopatológico del espécimen obtenido. Se puso énfasis en el aborto provocado como medio de control de la natalidad.

\section{Análiiss de los resultados}

Como puede observarse en el Cuadro № 1 , en el $23,4 \%$ de los casos se estableció el uso de maniobras abortivas por confesión de la paciente 0 por signos evidentes de las mismas, con la salvedad de que, en aborto sin causa aparente o traumáticos, seguramente se encierre algún porcentaje oculto de este tipo de aborto criminal por razón del silencio que guardan este tipo de pacientes aún en estado preagónico. El ab̌orto espontáneo sin causa aparente fue el de mayor incidencia con un 59,7\%.

\section{CUADRO № 1}

CLASE DE ABORTO

\begin{tabular}{lcr}
\hline Clase de Aborto & $\mathbf{N}^{\mathbf{0}}$ Casos & $\%$ \\
\hline Sin causa aparente & 418 & 59,7 \\
Traumáticos & 95 & 13,6 \\
Provocados & 164 & 23,4 \\
Habituales & 14 & 2,0 \\
Molares & 9 & 1,3 \\
$\quad$ TOTALES & 700 & 100,0 \\
\hline
\end{tabular}

En el Cuadro № 2, en lo que se refiere a la edad, el grupo de la tercera década de la vida arrojó la mayor frecuencia de abortos, tanto es-

CUADRO № 2

ABORTO PROVOCADO $Y$ GRUPO DE EDADES

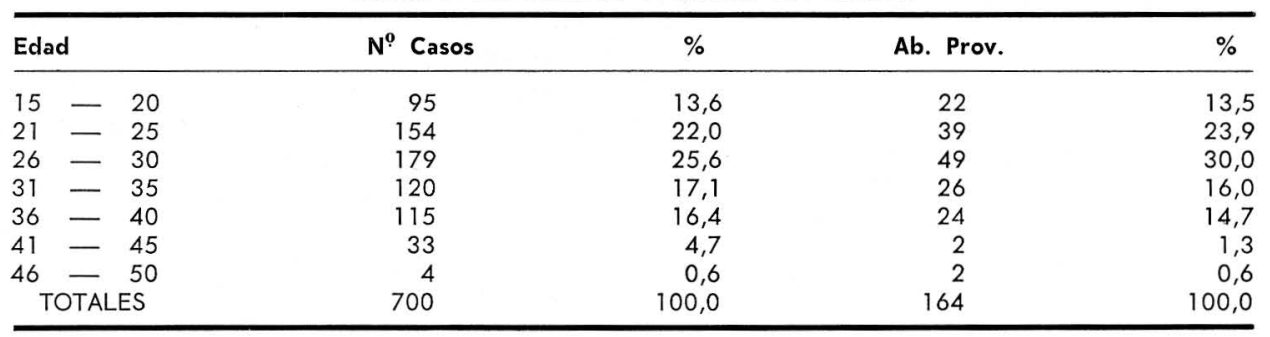

CUADRO NN 3

ABORTO PROVOCADO Y ESTADO CIVIL

\begin{tabular}{lcrrr}
\hline Estado civil & N $^{\mathbf{0}}$ Casos & $\%$ & Ab. Prov. & $\%$ \\
\hline Solteras & 354 & 50,6 & 97 & 59,1 \\
Casadas & 343 & 49,0 & 66 & 40,2 \\
Viudas & 3 & 0,4 & 1 & 0,7 \\
$\quad$ TOTALES & 700 & 100,0 & 164 & 100,0 \\
\hline
\end{tabular}


pontáneos $(64,7 \%)$, como provocados $(69,3 \%)$, que como se sabe es la de mayor actividad sexual. Las edades extremas fueron de 15 y 47 años.

En lo referente al estado civil (Cuadro № 3), la incidencia entre solteras y casadas, del aborto en general, es sensiblemente igual. Pero, en relación a los abortos provocados, las solteras presentan un índice mayor correspondiente al 59,1\% en comparación con las casadas que tiene un índice de 40,2\%.

En el Cuadro № 4 se relaciona la incidencia de abortos provocados con el grado de instrucción de la paciente. Es de notar que la mayor inci-

\section{CUADRO N: 4}

ABORTO PROVOCADO Y GRADO DE INSTRUCCION

\begin{tabular}{lcrrr}
\hline Instrucción & No Casos & $\%$ & Ab. Prov. & $\%$ \\
\hline Primaria & 574 & 82,0 & 133 & 81,1 \\
Secundaria & 32 & 4,6 & 10 & 6,1 \\
Analfabeta & 50 & 7,2 & 21 & 12,8 \\
No establecida & 43 & 6,2 & 0 & 0,0 \\
TOTALES & 700 & 100,0 & 164 & 100,0 \\
\hline
\end{tabular}

\section{CUADRO N: 5}

ABORTO PROVOCADO Y PARIDAD

\begin{tabular}{lccrr}
\hline Paridad & No Casos & $\%$ & Ab. Prov. & $\%$ \\
\hline Multíparas & 628 & 89,7 & 153 & 83,2 \\
Primíparas & 72 & 10,3 & 11 & 6,8 \\
$\quad$ TOTALES & 700 & 100,0 & 164 & 100,0 \\
\hline
\end{tabular}

dencia correspondió a las de educación primaria con un $82 \%$ de la totalidad de los abortos y con un $81,1 \%$ en relación a los abortos provocados. Las pacientes con instsrucción secundaria fueron las que tuvieron la más baja incidencia con un $6,1 \%$.
La mayor frecuencia de abortos provocados se registró en las multíparas con el $93,2 \%$, en tanto que en las primíparas la frecuencia fue baja con un 6,8\% (Cuadro № 5). Estos datos guardan relación con los encontrados en el trabajo sobre "Infección Pelviana" elaborado por nos-

\section{CUADRO № 6}

ABORTO PROVOCADO Y RAZA

\begin{tabular}{lcrrr}
\hline Raza & $\mathbf{N}^{\mathbf{0}}$ Casos & $\%$ & Ab. Prov. & $\%$ \\
\hline Blanca & 105 & 15,0 & 20 & 12,2 \\
Mestiza & 550 & 78,6 & 131 & 79,9 \\
Negra & 38 & 5,4 & 9 & 5,5 \\
No establecida & 7 & 1,0 & 4 & 2,4 \\
TOTALES & 700 & 100,0 & 164 & 100,0 \\
\hline
\end{tabular}


otros en la misma institución (4). A medida que la paridad aumenta es más frecuente la interrupción de la gestación por maniobras criminales. Estos datos coinciden con los registrados por otros autores nacionales (3).

En el Cuadro № 6 se observa que la mayor frecuencia de abortos correspondió a la raza mestiza con un $78,6 \%$; cabe anotar que, en esta región de la Costa, tanto la raza negra como la blanca son minoritarias. En lo que hace relación a la raza y el aborto provocado, correspondió a la mestiza el 79,9\%.

\section{CUADRO № 7}

SINTOMAS AL INGRESO

\begin{tabular}{|c|c|c|c|}
\hline Sintomas & No & Casos & $\%$ \\
\hline Metrorragia & & 659 & 94,1 \\
\hline Dolor & & 616 & 88,0 \\
\hline Fiebre & & 176 & 25,1 \\
\hline
\end{tabular}

En el Cuadro № 7 se anotan los síntomas más frecuentemente hallados como fueron la metrorragia, el dolor y la fiebre. Solo el $25,1 \%$ registró temperatura al ingreso.

CUADRO N: 8

\section{CONDICIONES GENITALES}

\begin{tabular}{|c|c|c|}
\hline Condiciones genitales & No Casos & $\%$ \\
\hline Ca. cervical & 1 & 0,1 \\
\hline Cervicitis & 51 & 7,3 \\
\hline Desgarros cervicales & 7 & 11,0 \\
\hline Desviaciones & 40 & 5,7 \\
\hline Prolapso genital & 1 & 0,1 \\
\hline Pólipos cervicales & 3 & 0,4 \\
\hline Trauma cervical & 1 & 0,1 \\
\hline TOTALES & 174 & 24,7 \\
\hline
\end{tabular}

En el Cuadro № 8 se determina la patología genital que corresponde a 174 casos de los 700 estudiados. El mayor porcentaje lo determinó la patología cervical con $11 \%$ de desgarros, $7,3 \%$ de cervicitis, un caso de Ca. de cérvix, etc. Siguió en su orden los vicios de posición uterina con $5,7 \%$.

En el Cuadro № 9 se observa que el $42,2 \%$ de este grupo de pacientes registró abortos anteriores.

\section{CUADRO № 9}

\section{ANTECEDE.NTES GINECO-OBSTETRICOS}

\begin{tabular}{|c|c|c|c|}
\hline $\begin{array}{l}\text { Antecedentes } \\
\text { gineco-obstétricos }\end{array}$ & $\mathrm{N}^{0}$ & Casos & $\%$ \\
\hline Abortos & & 296 & 42,2 \\
\hline Ectópicos & & 1 & 0,2 \\
\hline Primigestantes & & 72 & 10,3 \\
\hline Multigestantes & & 628 & 89,7 \\
\hline
\end{tabular}

Los antecedentes quirúrgicos están consignados en el Cuadro NN 10. De los casos estudiados, solo en 59 de ellos hay datos en la historia clínica. El antecedente quirúrgico de mayor frecuencia fue el legrado uterino; le siguió la apendicectomía.

CUADRO Nํ 10

\section{ANTECEDENTES QUIRURGICOS}

\begin{tabular}{|c|c|}
\hline Intervención & $\mathrm{N}^{0}$ Casos \\
\hline Legrados Uterinos & 28 \\
\hline Apendicectomías & 9 \\
\hline Cesáreas & 5 \\
\hline Hernioplastias & 5 \\
\hline Quistes de ovario & 5 \\
\hline Colecistectomías & 3 \\
\hline Salpingectomía & 1 \\
\hline Colporrafia & 1 \\
\hline Sefenectomía & 1 \\
\hline Amigdalectomía & 1 \\
\hline TOTAL & 59 \\
\hline
\end{tabular}

En el Cuadro № 11 se muestra que el $45,8 \%$ confesó el deseo de no tener más hijos. Esa incidencia aumenta al tener en cuenta el grupo de pacientes indiferentes al deseo de tener hijos (20,5\%), actitud compati- 
ble con el rechazo a la maternidad. De las 321 pacientes que manifestaron el deseo de no tener más hijos, 192 confesaron que el motivo del rechazo a la maternidad lo constituye la difícil situación económica y el sentirse satisfechas con los hijos que tienen, como puede verse en el Cuadro N. 12.

\section{CUADRO N: 11}

DESEOS DE MAS GESTACIONES

\begin{tabular}{lcr}
\hline Deseos de procrear & No Casos & $\%$ \\
\hline No & 321 & 45,8 \\
Sí & 236 & 33,7 \\
Inciiferente & 15 & 2,1 \\
No establecido & 128 & 18,4 \\
\hline
\end{tabular}

\begin{tabular}{lr}
\multicolumn{3}{c}{ CUADRO No 12} \\
MOTIVOS PARA NO MAS GESTACIONES \\
\hline Motivos & No Casos \\
\hline Mala situación económica & 99 \\
Tiene muchos o suficientes & 61 \\
Miedo a la gestación o parto & 17 \\
Abandono del marido & 10 \\
Edad avanzada & 4 \\
No ser casada & 1 \\
TOTALES & 192 \\
\hline
\end{tabular}

Con relación al ingreso del grupo de pacientes investigados, solo 424 de ellas suministraron la información correspondiente. Los ingresos mensuales variaron desde 30 hasta 1.200 pesos. De esta última cifra solo se encontraron 3 casos. El promedio de ingresos, tanto para las de aborto provocado como para las de aborto espontáneo, fue de 300 a 450 pesos.

En el Cuadro № 13 relacionado con la nutrición, se anota que el $83 \%$ tiene un tipo de nutrición regular, compatible con el grado social de las pacientes que acuden a esta institución y deficiente en el resto de los casos.
CUADRO NN: 13

ESTADO NUTRICIONAL

\begin{tabular}{|c|c|c|}
\hline Nutrición & $N^{0}$ Casos & $\%$ \\
\hline Suficiente & 72 & 10,3 \\
\hline Regular & 581 & 83,0 \\
\hline Mala & 47 & 6,7 \\
\hline TOTALES & 700 & 100,0 \\
\hline
\end{tabular}

En el Cuadro № 14 relacionado con la profesión, vale la pena destacar que el mayor grupo de estas pacientes correspondió a las de Oficios Domésticos con el 97,3\%, hecho explicable por razón de sus condiciones económicas de su numerosa familia.

\section{CUADRO № 14}

PROFESION

\begin{tabular}{|c|c|c|}
\hline Profesión & $\mathrm{N}^{0}$ Casos & $\%$ \\
\hline Oficios domésticos & 681 & 97,3 \\
\hline Obrera & 9 & 1,3 \\
\hline Lavadora & 6 & 0,8 \\
\hline Mesera & 2 & 0,3 \\
\hline Comerciante & 1 & 0,1 \\
\hline Secretaria & 1 & 0,1 \\
\hline
\end{tabular}

El Cuadro № 15 muestra los hallazgos anatomopatológicos. Del grupo de pacientes investigados se hizo el estudio histopatológico en 656 casos; en 117 casos no se demostró evidencia de embarazo histológicamente. En el grupo que reveló embarazo hubo infección en 158 casos; en 111 casos se registraron cambios de Arias Stella y en 49 casos de Molas, 30 correspondieron a Mola incipiente. En el resto de casos de no embarazadas conviene anotar la alta incidencia de endometrio proliferativo, un caso de $\mathrm{Ca}$. de cérvix estado II y un caso compatible con lesiones luéticas placentarias. 
CUADRO № 15

ESTUDIO HISTOPATOLOGICO

\begin{tabular}{lrr}
\hline Hallazgos histológicos & No Casos & $\%$ \\
\hline Restos ovulares infectados & 158 & 22,6 \\
Restos ovulares no infectados & 367 & 52,4 \\
Vellosidades hidrópicas & 30 & 4,3 \\
Cambios de Arias Stella & 111 & 15,8 \\
Mola & 19 & 2,7 \\
Endometritis & 43 & 6,1 \\
Endometritis sincicial & 1 & 0,1 \\
Endometrio proliferativo & 40 & 5,7 \\
Endometrio secretorio & 8 & 1,1 \\
Hiperplasia endometrial & 3 & 0,4 \\
Sangre coagulada & 6 & 0,9 \\
Pólipo endometrial & 3 & 0,4 \\
Pólipo cervical & 2 & 0,3 \\
Restos de leiomioma & 1 & 0,1 \\
Cervicitis & 4 & 0,7 \\
Lesiones luéticas placentarias & 1 & 0,1 \\
Ca. cervical & 1 & 0,1 \\
TOTAL & 656 & \\
\hline
\end{tabular}

\section{Comentarios}

La incidencia de aborto provocado como control de la natalidad es una realidad escandalosa en nuestro medio que produce una alta incidencia de morbi-mortalidad materna como ha sido registrado en nuestro estudio sobre Infección Pelviana (4). Este recurso como control de la natalidad revela una falta de responsabilidad, de cultura, educación religiosa y relajamiento moral de un grupo de nuestra población que se encuentra a merced de organizaciones especializadas en estos menesteres por falta de una legislación más estricta y severa sobre el aborto criminal. En algunos países creyeron darle solución a este problema legalizando el aborto criminal con resultados desalentadores que han determinado la modificación de dicha conducta, dando una mayor ayuda oficial y protección al matrimonio con familias numerosas.

El aborto criminal en nuestro estudio registró una mayor frecuencia en la tercera década de la vida, etapa considerada como de mayor actividad sexual y mayor registro de embarazos. Correspondió también la mayor incidencia de abortos provocados en las mujeres solteras, de nutrición regular, de bajo nivel de instrucción y bajos ingresos económicos; factores sociales estos que tienen gran importancia en la interrupción de su embarazo por razón de que este gran núcleo de la población no encuentra protección de parte del Estado, ni de parte de sus hijos. Se registró en este trabajo que el $66,3 \%$ de las pacientes confesaron el rechazo de su embarazo por razones de tipo económico y de una paternidad irresponsable. En este mismo grupo se encontró que la entrada mensual promedio oscilaba entre 300 y 450 pesos, razón que apoya los motivos anteriores confesados por las pacientes para el rechazo de su embarazo. El $83 \%$ de estas pacientes reveló también un estado nutricional regular, por no decir deficiente.

En lo que hace al examen histopatológico es bueno aclarar que el material estudiado se obtuvo en su totalidad del espécimen obtenido del legrado uterino. Se comprobó el embarazo en el $82,2 \%$; de estos casos de embarazo se registraron 30 con degeneración hidrópica de las vellosidades coriónicas, cambios histopatológicos relacionados con la Mola Hidatiforme $(5,6,7)$ y catalogados como mola incipiente y que sumados a los 19 casos de Mola hidatiforme arroja una incidencia de mola de $9 \%$. Lo anterior da una relación de 1 mola por cada 11 embarazos, incidencia considerablemente alta. La infección se encontró en 29,3\%, todos clínicamente asintomáticos. En el grupo de pacientes en donde no se comprobó embarazo se registró una alta incidencia de endometrio de tipo proliferativo. 
El aborto criminal sigue siendo una gran causa de pérdida de vidas humanas $(8,9)$, especialmente en la clase de condiciones socio-económicas, morales y religiosas de bajo nivel; sin embargo, se abren nuevos rumbos y esperanzas con las últimas orientaciones dadas por las jerarquías de la Iglesia Católica, especialmente consignadas en la reciente Encíclica "Populorum Progresio" de la que me permito tomar los siguientes conceptos sobre planificación familiar: "Es verdad que con demasiada frecuencia un crecimiento demográfico acelerado añade sus dificultades intrínsecas a los problemas del desarrollo; el tamaño de la población crece más de prisa que los recursos disponibles, y parece que se hubiere llegado a un callejón sin salida. En este momento es muy grande la tentación de frenar el aumento demográfico con medidas radicales. No hay duda de que las autoridades públicas pueden intervenir, dentro de los límites de su competencia, al facilitar el acceso a la información apropiada y al adoptar medidas convenientes siempre que éstas estén en conformidad con la ley moral y que respeten la legítima libertad de los esposos. Cuando desaparece el derecho inalienable al matrimonio y a la procreación, deja de existir la dignidad humana. Por último, concierne a los padres decidir, con pleno conocimiento del asunto, el número de hijos, tomando en cuenta sus responsabilidades ante Dios, ante sí mismos, ante los hijos que ya hayan traído al mundo y ante la comunidad a que pertenecen. En todo esto deben seguirse los dictados de su propia conciencia guiada por la Ley' Divina auténticamente interpretada, $y$ sostenida por la confianza en El".

En esta declaración la Iglesia acepta los derechos de todo gobierno pa- ra organizar programas y suministrar enseñanza sobre la planificcaión de la familia, respetando el derecho de cada pareja para regular el número de hijos que desee tener y el método que quieran usar dentro de los dictados de su moral. La ausencia de la no planificación de la familia ha sido una de las causas mayores del aborto criminal. Este hecho se ha comprobado con la disminución del aborto provocado en los países cuyos gobiernos han implantado oficialmente la planificación de la familia. Abrigamos la esperanza de que entre nosotros, en un futuro inmediato, se pueda registrar la disminución de este problema social con la autorización por parte del Estado en colaboración con las autoridades eclesiástisas, de métodos de planificación familiar que hagan efectiva la paternidad responsable.

\section{Resumen}

1. Se hizo una selección de 700 historias clínicas de pacientes ingresados a la sección de abortos con una clasificación relacionada con el estado civil, estado nutricional, edad, ra$z a$, antecedentes obstétricos y quirúrgicos, grado de instrucción, situaciórı económica y deseo de tener hijos.

2. De la serie estudiada, el 23,4\% confesaron haberse provocado el aborto. Correspondió a la tercera década de la vida, a las solteras, al bajo nivel de instrucción, a la difícil situación económica y al mal estado nutricional la mayor frecuencia de este tipo de abortos.

3. Correspondió a las multíparas su mayor incidencia.

4. La patología genital más frecuentemente encontrada fue la de tipo cervical. 
5. El $66,3 \%$ de las pacientes confesaron el deseo del rechazo a su embarazo o a no tener más hijos, justificando su actitud con la mala situación económica.

6. El estudio histopatológico reveló una alta incidencia de Mola con un $9 \%$.

\section{Summary}

1. Seven hundred clinical histories from abortion patients were classified according to civil status, nutritional state, age, race, obstetrical and surgical history, education level, economical situation and desire to have children.

2. $23.4 \%$ frmo this series confessed provoked abortion. The majority of patients with this type of abortion were in their thirties, single, with a low education level, difficult economic situation and in bad nutritional state.

3. The higher incidence was found among the multiparous.

4. The genital pathology found with higher frequency was of the cervical type.
5. $66.3 \%$ of the patients admitted the wish for no more pregnancies, mostly due to their bad economic situation.

6. Histopathological study showed a high mola incidence, up to $9 \%$.

\section{BIBLIOGRAFIA}

1 ANDRAS KLINGER: Hungarian Central Office of Stolistices Family Planning and Population Programs. Chicago University Press. 1964.

2 KATISCH R. V.: Rev. Inmun. 30: 45, 1966.

3 SANTAMARIA PAEZ L. E.: Aborto Séptico y Maniobras Abortivas. Rev. Col. Obst. y Ginec. 17: 273, 1968.

4 ACOSTA BENDEK E., DUVA PALACIO J.: Infección Pelviana. Rev. Col. Obst. y Ginec. 19: 29, 1968.

5 HERTIG A., EDMONDS H. W.: Genesis of Hydatidiform Mole. Arch. Path. 30: 260, 1940.

6 NOVAK E.: Gynecology and Obstetric Pathology. Saunders Company. Philadelphia. 1953.

7 HUBER C. P., y cols.: Changes in Chorionic Tissue of Aborted Pregnancy. Am. J. Obst. \& Gynec. 73: 569, 1957.

8 MORITZ G. R., THOMPSON N. S. Septic Abortion. Am. J. Obst. \& Gynec. 6: 851, 1963. 